Transparenz und Reputation als Basis never Steuerungsstrukturen

\section{Hin zur Sustainability Governance}

\begin{abstract}
Die Beziehungen zwischen Gesellschaft und Natur waren über lange Zeit eher durch eine relativ enge Interaktion geprägt. Störungen machten sich direkt bemerkbar und haben quasi "natürlich" zu Reaktionen - oder zur Wahrnehmung der "exit option" - geführt. Die so genannte tragedy of the commons erweist sich in Wirklichkeit als Problem des unbeschränkten Zugangs. Normen und Regeln der Bewirtschaftung der Commons haben sich bei globalen Umweltproblemen aber noch nicht entwickelt. Hierin liegt das Kernproblem, das eine nachhaltigkeitsorientierte Governance lösen muss.
\end{abstract}

$\mathrm{N}$ Von Ulrich Petschow und Jens Clausen Umweltprobleme wie Ozonloch und Klimaproblematik sind dadurch gekennzeichnet, dass sie nicht mehr direkt wahrnehmbar sind und daher letztlich über wissenschaftliche Erkenntnisse und Modellbildungen erst vermittelt werden müssen. Auch ist fest zu stellen, dass diese schleichenden Degradierungen nicht mehr zwangsläufig auf der lokalräumlichen Ebene anfallen, sondern in zum Teil komplexen Wirkungsmechanismen auf globaler Ebene wirksam werden, und von daher kollektives Handeln mit Blick auf diese Ebene erfordern. Mit diesem Maßstabswechsel der Problemlagen ist verbunden, dass die Problemlösungsansätze ungleich komplexer geworden sind, nicht zuletzt durch die enge Verknüpfung mit intra- und intergenerativen Verteilungsfragen.

Gleichwohl existieren erhebliche Meinungsunterschiede dahingehend, wie die Situation des open access überwunden werden kann: Auf der einen Seite wird im Sinne einer vertieften Integration ein globales Management der Umweltressourcen gefordert. Auf der anderen Seite werden im Sinne einer Dezentralisierung und auch Regionalisierung gemäß dem Subsidiaritätsprinzip eher Regelungen zur Ausgestaltung der regionalen bzw. 10kalen Lebensgemeinschaften für erforderlich gehalten, die diese Übernutzung auch global verhindern. Damit stellt sich die Frage, was letztlich geeigneter ist, einen Beitrag zur Sicherung der Global Commons zu leisten: eine top-downStrategie, die auf Rahmenentwicklung im Sinne von Government hinausläuft, oder eine bottomup-Strategie, die auf die Verantwortungsverlagerung auf dezentrale Akteure zielt (1).

\section{Globalisierung von Steuerungs- strukfuren}

Zeitgleich mit den benannten Problemlagen ist fest zu stellen, dass die herkömmlichen Steuerungsstrukturen, die auf den Nationalstaat als zentrale Instanz fokussieren, aus zumindest zwei Gründen an Grenzen gestoßen sind: Zum einen sind bereits in den 70er Jahren die Grenzen des Planungsstaates hinsichtlich seiner Fähigkeiten zur Feinsteuerung aufgezeigt worden; zum anderen wird im Zuge der Globalisierungsdiskussion deutlich, dass das zunehmende Auseinanderfallen zwischen ökonomischer und ökologischer Problem- und Steuerungsebene veränderte Steuerungsformen erforderlich macht. Beides zusammen bedeutet, dass sowohl grundsätzlich eine Rückgabe von Kompetenzen auf untere Ebenen als auch eine Abgabe von Kompetenzen nach oben erforderlich ist. Eng damit verbunden ist die Einbeziehung weiterer Akteure, sowohl auf der lokalen als auch der globalen Ebene.

Zugleich verändert sich staatliche Autorität. Diese kann weniger im Alleingang aktiv werden, sondern muss zunehmend in Kooperationen eintreten, beispielsweise bei der Lösung grenzüberschreitender Probleme, und ist zugleich nicht mehr zwangsläufig unangefochten.

Die internationale Kooperation von Staaten findet bislang im Wesentlichen im Rahmen spezifischer internationaler Organisationen und Regime statt. Die Transparenz der Entscheidungsstrukturen erweist sich dabei vielfach als begrenzt. Diese „Club“-Strukturen werden nach Keohane/ Nye (2) zunehmend in Frage gestellt, indem 1. nicht-staatliche Akteure wie Unternehmen, Verbände, Gewerkschaften und Nichtregierungsorganisationen (NGOs) Einfluss gewinnen,
2. die Segmentierung der Regime herausgefordert wird und interlinkages, wie etwa zwischen Welthandelsorganisation und Umwelt, betont werden, nicht zuletzt durch neue Akteure in diesen Arenen, und

3. auf das Demokratie- oder zumindest Legitimitätsdefizit dieser Regime verwiesen wird.

Diese veränderten Formen der Steuerung, in denen staatliche Autorität eine wichtige, gleichwohl nicht mehr zwangsläufig zentrale Rolle spielt, sowie die Herausbildung von ,,spheres of authority" (3), die vielfach nicht mehr mit den formalen Rollenzuweisungen verbunden sind, werden unter dem Begriff Governance gefasst. Governance hat dabei per se weder eine positive noch eine negative Konnotation, diese wäre letztlich erst am Steuerungsergebnis in dem jeweiligen Feld zu bewerten.

Die Überwindung des Demokratiedefizits im globalen Raum ist gegenwärtig nur schwerlich vorstellbar, dies ist selbst im Rahmen der Europäischen Union, nicht zuletzt aufgrund einer begrenzt entwickelten europäischen Öffentlichkeit, nicht sichergestellt. Gleichwohl kann ein Legitimationsgewinn durch Transparenz und Zurechnung von Verantwortlichkeit erreicht werden. Dabei sind unterschiedliche Elemente zentral:

1. Die Schaffung einer Öffentlichkeit, unter anderem via NGOs und Medien,

2. Transnationale Netzwerke von Berufs- aber auch Interessengruppen,

3. Märkte, die zumindest durch Kundenreaktionen Wirkungen zeitigen können, aber auch Ratingagenturen, sowie

4. Codes of Conduct unterschiedlichster Organisationen.

Ansätze in dieser Richtung sind erstens die unterschiedlichen, insbesondere trisektoralen Netzwerke (4, vgl. auch den Beitrag von Streck), die hybride Formen der Governance darstellen. Ihr Erfolg oder Misserfolg hängt zentral von der Transparenz aber auch der generellen Akzeptanz der von ihnen erzielten Ergebnisse ab. Ein zweiter Ansatz ist die Schaffung neuer Formen der Transparenz und Verantwortlichkeit von internationalen Regierungsorganisationen, NGOs als auch von Unternehmen, um die Legitimitätslücken zu schließen.

Wesentliche Veränderungen, die insbesondere seit den 80er Jahren zu beobachten sind, sind auf der eher ökonomischen Ebene die zunehmende Liberalisierung und Marktöffnung in den jeweiligen Staaten. Diese Veränderungen haben unter anderem zur Konsequenz, dass marktwirtschaftliche 
Steuerungslogiken an Bedeutung gewinnen. Diese Logiken - und damit marktlich vermittelte Formen des Austausches - haben sich in unterschiedlichem Ausmaß in allen Ländern durchgesetzt.

\section{- Private Regulierungsformen}

Die Globalisierung der ökonomischen Aktivitäten hat - nicht allein auf die Umwelt bezogen - einen mismatch zwischen Märkten und Politiken entstehen lassen. Eine Reaktion von Unternehmen ist die Konstruktion von privaten internationalen Regimes als eine Form der Selbstregulierung oder Regelsetzung in Abwesenheit eines übergreifenden globalen politischen Systems (5). Die Kooperationsansätze nehmen dabei vielfältige Formen an, von informellen Industrienormen, koordinierenden Dienstleistungsunternehmen bis hin zur Bildung transnationaler Unternehmensverbände (vgl. auch den Beitrag von Schneider/Ronit). Wesentliche Motive sind

die Steigerung der Effizienz der Markttransaktionen,

- die Stabilität und Sicherheit der Transaktionen, aber auch

- die Reaktion auf Anforderungen der Stakeholder, aus Gründen der Reputation aber auch um durch Codes of Conduct etc. staatliche Regulierungen vorweg zu nehmen oder abzuwenden.

\section{- Legitimation von Selbstregulierung}

Arrangements der Selbstregulierung haben in den letzten zwei Jahrzehnten auf den unterschiedlichen Ebenen deutlich zugenommen, insbesondere auch im Hinblick auf die Regulierung der Multinationalen Unternehmen. Exemplarisch kann dies anhand der OECD Guidelines für multinationale Unternehmen aufgezeigt werden, deren Ziel es 1976 letztlich war, politische Regulierungen durch freiwillige Selbstverpflichtungen zu vermeiden. Diese Guidelines wurden mehrfach überarbeitet und dabei Themenfelder aufgenommen, die in der gesellschaftlichen Diskussion eine zunehmende Rolle spielen. Ihre letzte „Renovierung“ im Jahre 2000 beinhaltete weitgehende Richtlinien für das Umweltverhalten der Unternehmen, gleichwohl konnte eine Berichtspflicht nicht verankert werden. Damit ist eine Überprüfbarkeit weiterhin nicht gegeben. Auf Branchenebene können die Möglichkeiten und Grenzen dieser Selbstregulierung am Beispiel der Responsible Care Kampagne der Chemischen Industrie aufgezeigt werden. Diese wurde in der Folge des Reputationsverlustes durch eine Reihe von verheerenden Unglücken initiiert, um das
Image der Chemischen Industrie zu verbessern und weiter gehende gesetzliche Regulierungen zu vermeiden. Der Erfolg solcher Kampagnen ist zum Teil durchaus bemerkenswert, allerdings werden zunehmend auch Zweifel laut. So kommt eine Studie von King et al. zu dem Ergebnis, dass US-Unternehmen, die an der Responsible Care Kampagne teilnehmen, aus Umweltsicht keineswegs über eine überdurchschnittliche Performance verfügen (6). Überprïft wurde dies anhand der Toxic Release Inventories in den USA, die eine Veröffentlichung der Daten bestimmter Gefahrstoffe vorschreiben und öffentlich zugänglich sind.

Dies deutet darauf hin, dass es den Verbänden nur begrenzt gelingt, ihre Mitglieder zu verpflichten und das Reputationsziel zu erreichen. Wie an dem Beispiel deutlich wird, ist die Informationsverfuigbarkeit zentral für die Überprüfung der jeweiligen Selbstregulierungsmechanismen. Ohne die öffentliche Verfügbarkeit der Daten wäre ein solcher Vergleich von Unternehmen nicht möglich und ein direkter Nachweis von Umsetzungsproblemen daher auch nicht nachvollziehbar gewesen. Die Überprüfbarkeit ist aber ein Essential der Glaubwürdigkeit von solchen Selbstverpflichtungen.

Die Überprüfung entsprechender Codes wird allerdings von einigen besonders im Rampenlicht stehenden und um ihre Reputation besorgten Unternehmen mittlerweile zugestanden. Hier ist insbesondere das Beispiel der Firma Nike anzuführen, die die Einhaltung ihrer Codes an den jeweiligen Standorten von externen ,Sachverständigen“ sprich NGOs überprüfen lässt. Es kann an dieser Stelle nicht auf die Kriterien eingegangen werden, gleichwohl ist darauf zu verweisen, dass es durchaus Probleme im Hinblick auf die Auswahl der Kontrolleure gibt. Denn es besteht ebenfalls eine Konkurrenz zwischen den unterschiedlichen zivilgesellschaftlichen Akteuren; damit stellt sich auch für diese die Legitimationsfrage.

\section{Probleme der Commons heute}

Zentral für das Problem kollektiver Handlungen ist die fehlende Kongruenz zwischen den individuellen Anreizsystemen und dem gewünschten Ergebnis für die (Welt-) Gemeinschaft.

Die beobachtbare Dispersion von Macht und Verantwortlichkeit stellt neue Herausforderungen an die - auch prozedurale - Legitimität von Entscheidungs- und Verhaltensweisen der unterschiedlichen Akteure. Hieraus ergeben sich zentrale Anforderungen an die Transparenz und Verantwortungszuweisung. Gerade auch im Hinblick auf Handlungen vor dem Hintergrund von Nachhaltig- keitszielen ist dabei der Aspekt der Reputation wesentlich, der vor dem Hintergrund der wiederholten Interaktionen zwischen den Akteuren an Bedeutung gewinnt. Er steht in engem Zusammenhang mit Rechenschaftspflichten und Sanktionsmöglichkeiten (naming und shaming).

Zusammenfassend bedeutet dies, dass die Verantwortlichkeit sämtlicher Akteure in den Governance-Netzwerken und -Strukturen im Sinne der Nachhaltigkeit entwickelt werden muss. Dies läuft letztlich zentral auf die Publizität zentraler Informationen und ihre Bewertung, nicht allein durch zivilgesellschaftliche Akteure, hinaus. Die bestehende Transparenz auf den Kapitalmarkt, etwa durch Kreditratingagenturen, und die damit angestrebte Überwindung des Prinzipal-AgentenProblems im Rahmen der Corporate Governance sollte daher eine Entsprechung im Hinblick auf das Naturkapital in Form einer Sustainability Governance finden.

\section{Anmerkungen}

(1) Vgl. Paterson, M.: Understanding Global Environmental Politics. Domination, Accumulation, Resistance. Houndmills 2000 .

(2) Keohane, R.0./ Nye, J.S.: The Club Model of Multilateral Cooperation and Problems of Democratic Legitimacy. 2000.

(3) Rosenau, J.N.: Towards an Ontology for Global Governance. In: Hewson, M./ Sinlaird, T.J. (eds.): Approaches to Global Governance Theory. New York 1999.

(4) Reinicke, W.H.: Global Public Policy. Governing without Government? New York 1998.

(5) Vgl. Haufler, V.: Private sector international regimes. In: Higgott, R.A. et al. (eds.): Non-State Actors and Authority in the Global System. London 2000. Insbesondere das Verhältnis von Unternehmen und NGOs in solchen Regimen wurde vom IÖW im Rahmen der BMBF-Sondierungsstudie „Die Zivilgesellschaft als Akteur der Unternehmenssteuerung im Rahmen der Global Governance" vertieft untersucht. Eine Veröffentlichung ist in Vorbereitung.

(6) King, A./ Lenox M.: Industry self-regulation without sanctions: The chemical industry's responsible care program. In: Academy of Management Journal, Vol. 43(2000), №. 4.

\section{Die Autoren}

Ulrich Petschow und Jens Clausen sind wissenschaftliche Mitarbeiter des Instituts für ökologische Wirtschaftsforschung.

Kontakt: IÖW, Potsdamer Str. 105, 10785 Berlin. Tel. 030/ 884594-0, Fax 030/ 8825439, E-mail: ulrich.petschow@ioew.de, jens.clausen@hannover.ioew.de 
(c) 20I0 Authors; licensee IÖW and oekom verlag. This is an article distributed under the terms of the Creative Commons Attribution Non-Commercial No Derivates License (http://creativecommons.org/licenses/by-nc-nd/3.o/), which permits unrestricted use, distribution, and reproduction in any medium, provided the original work is properly cited. 